"This is the peer reviewed version of the following article: [Termination of DNA Replication in Prokaryotes], which has been published in final form at [https://doi.org/10.1002/9780470015902.a0001056.pub3] This article may be used for non-commercial purposes in accordance with Wiley Terms and Conditions for Self-Archiving 
eLS

\section{Termination of DNA Replication in Prokaryotes}

Christian J. Rudolph ${ }^{1}$

Tayla-Ann Corocher ${ }^{2,3}$

Ian Grainge ${ }^{2}$

*Iain G. Duggin ${ }^{3}$

${ }^{1}$ Division of Biosciences, College of Health and Life Sciences, Brunel University London, Uxbridge, UB8 3PH, United Kingdom.

${ }^{2}$ Faculty of Science, School of Environmental and Life Sciences, University of Newcastle, Callaghan, NSW 2308, Australia

${ }^{3}$ The ithree institute, University of Technology Sydney, NSW, 2007, Australia

*Correspondence: Iain.Duggin@uts.edu.au

Published Online:

Advanced Article

(Advanced articles are aimed at advanced undergraduates, graduate students, postgraduates, and researchers reading outside their field of expertise.)

\section{Abstract}

Most bacteria and archaea have circular chromosomes, in which DNA replication begins at a site known as an origin of replication. Double-stranded DNA unwound at the origin creates two replication forks that are engaged by DNA polymerase complexes (replisomes) that advance each fork and proceed in opposite directions away from the origin, copying the original strands. Termination of DNA replication occurs when the two forks meet and fuse, creating two separate double-stranded DNA molecules. In the well-studied bacteria Escherichia coli and Bacillus subtilis, this occurs in the terminus region, which is situated diametrically opposite the origin. Failure to terminate chromosome replication correctly can lead to problems with genome function and stability, including DNA over-replication. In contrast, some archaea have multi-origin chromosomes and do not appear to specifically regulate the location of termination.

\section{Keywords:}


- DNA replication;

- replication termination;

- replication fork arrest;

- DNA terminators;

- replication terminator protein;

- termination utilization substance;

- catenanes

\section{Key Concepts:}

- Termination of DNA replication occurs when two oppositely-orientated replication forks meet and fuse, to create two separate and complete double-stranded DNA molecules.

- In circular bacterial chromosomes, termination is restricted to a region called the terminus region, located approximately opposite the origin of replication.

- A replication fork trap is an opposing arrangement of unidirectional replication terminator (Ter) sites in a region of DNA, which allows replication forks to enter the trap from either direction, but not exit it.

- Failure to terminate bacterial chromosome replication correctly results in chromosome overreplication and genome instability.

\section{Introduction}

Chromosome replication and the cell cycle of bacteria such as Escherichia coli and Bacillus subtilis shows significant differences to that of eukaryotic cells. Whereas eukaryotes typically have multiple linear chromosomes with cell cycle events are temporally distinct and under the control of strict checkpoint systems, these bacteria have a single circular chromosome ( 4-5 Mbp) and exhibit overlapping cell cycle events. The DNA, which forms a compact so-called nucleoid inside the cell, is duplicated by two replication machineries, or replisomes, that start from a unique site called the origin of chromosome replication (oriC), and then move in opposite directions around the circular chromosome, processively replicating the DNA to create two identical chromosomes (Beattie and Reyes-Lamothe, 2015; Jameson and Wilkinson, 2017). The replicating DNA is continuously segregated towards opposite cell halves, while replication of the remainder of the chromosome is still ongoing (Reyes-Lamothe et al., 2012). Remarkably, many bacteria can also simultaneously carry out multiple rounds of chromosome replication, in order to replicate DNA fast enough during high growth rates, i.e. a new round of chromosome replication is started while previous rounds of duplication and segregation are still ongoing. In nutrient-rich conditions, this allows a single $E$. coli cell to have up to 3 division events per hour, even though it takes at least 40 minutes to complete replication of the chromosome (Helmstetter et al., 1968; Zaritsky and Woldringh, 2015). A pair replication forks originating together 
from oriC eventually approach one another and fuse within a region diametrically opposite oriC, called the terminus region. After termination, the two daughter chromosomes are further resolved through post-replicative processing if they remained interlinked or joined together as a chromosome dimer (Lesterlin et al., 2004; Reyes-Lamothe et al., 2012). These processes are temporally and spatially coordinated with the formation of a cell division septum at mid-cell between the two daughter chromosomes (Reyes-Lamothe et al., 2012; Zaritsky and Woldringh, 2015).

The emphasis in this review will be on the approach and fusion of the forks in the terminus region, the importance of its regulation, and how termination relates to post-replicative chromosome resolution.

\section{The bacterial chromosome terminus region and replication fork trap}

In E. coli and B. subtilis, the location of fork fusion is restricted to the centre of the terminus region by several replication pause sites (explained further below). In contrast, in diverse eukaryotes and archaea that have multiple replication origins per chromosome, fork fusion does not appear to be controlled by specific pause sites in many cases and instead occurs at random sites within fork fusion zones between origins (Duggin et al., 2011; Gambus, 2017; Hawkins et al., 2013; Samson et al., 2013).

The terminus region in both E. coli and B. subtilis functions as a "replication fork trap". This is due to the presence of a series of short DNA sequences, each having the potential to pause replication fork progression. Each of these so-called DNA terminator (or Ter) sites is asymmetric; it can arrest a fork approaching from one direction but not the other. The positioning and orientation of the terminators creates two opposed groups that would allow forks to enter but not exit the terminus regions, as shown in Figure 1. In E. coli the identified terminators are distributed over $42.5 \%$ of the chromosome, whereas in $B$. subtilis the spread is much narrower $(9.9 \%)$. However, in E. coli only the inner-most 4 sites are substantially involved in DNA replication fork arrest during normal chromosome replication (Duggin and Bell, 2009), and these sites are therefore considered to constitute the primary fork trap (spanning $8.7 \%$ of the chromosome). Nevertheless, the two opposed subgroups of terminators flank the central portion of the terminus region, such that those in one subgroup (TerC, B, F, G and $J$ in $E$. coli) can arrest a clockwise-moving fork, and those in the other subgroup (TerA, D, E, I and $H$ in E. coli) can block a anticlockwise-moving fork. How the fork trap operates can be understood by considering, for example, the clockwise fork from oriC in E. coli; after initially passing through the permissive orientations of $\operatorname{TerH}, I, E, D$ and $A$, it will encounter blockage at $\operatorname{Ter} C$ and be arrested for a finite period (a few minutes or so). If the anticlockwise fork does not progress to meet the clockwise fork while the latter is stalled, and thus achieve termination of replication, the clockwise fork proceeds to meet TerB, where it stops again, and so on until fusion with the anticlockwise fork is finally achieved. A similar situation would arise if the anticlockwise fork were to arrive first (initially encountering TerA). Thus, 
the multiple terminators appear to function as backups to ensure that any fork entering the terminus region does not exit it. Because of the positioning of $\operatorname{TerC}$ relative to the origin of replication and the other terminators, it is generally the first to be encountered by a replication fork (the clockwise one) and is considered to be the most frequently used terminator site in E. coli (Duggin and Bell, 2009). Of the nine Ter sites in B. subtilis (Figure 1b), TerI at $172^{\circ}$ is generally the first to be encountered by a fork (the clockwise one) and is probably the most frequently used in this organism. In both B subtilis and E. coli, the outer terminators are probably used only rarely (Duggin and Bell, 2009; Griffiths and Wake, 2000).

\section{DNA Terminator (Ter) sites}

In order to arrest a replication fork, a DNA terminator must bind a specific terminator protein, Tus (terminus utilization substance) in E. coli, and RTP (replication terminator protein) in B. subtilis. The genes encoding these proteins (tus and $r t p$ ) are themselves located within the terminus region (Figure 1). Their expression is auto-regulated through binding of the terminator protein to upstream terminators (TerB in E. coli and both TerI and TerII in B. subtilis) located very close to the gene's promoter, negatively influencing transcription; this keeps the amount of the terminator proteins low in each cell (in the order of 100 copies per cell) (Ahn et al., 1993; Natarajan et al., 1991; Roecklein et al., 1991; Roecklein and Kuempel, 1992).

The Ter sites in E. coli (Figure 1a) were defined as a $23 \mathrm{bp}$ long sequence, but show strongest conservation and importance for Tus binding between residues 6 and 19 inclusive (Figure 1b) (CoskunAri and Hill, 1997). A single molecule (monomer) of Tus binds specifically to each sequence (Figure 2a). The B. subtilis Ter sequences are completely different to those in E. coli. B. subtilis Ter sites show conservation over a 28 bp long sequence (Figure 1d), and contain two overlapping binding sites - an 'A site' and a 'B site' - each of which is capable of binding a dimer of RTP (Figure 2b). Both the A and B sites must bind a dimer of RTP in order to confer fork arrest activity. However, only replication forks approaching the B-site end of the Ter sequence are arrested, accounting for the polarity of action (Smith and Wake, 1992). Interestingly, both naturally occurring and synthetic terminators exist on $B$. subtilis plasmids that are composed of two B sites instead of an A and a B site; these terminators have the ability to arrest DNA replication forks approaching from either direction (Meijer et al., 1996; Smith et al., 1996). An equivalent 'bidirectional' terminator has not been discovered in E. coli. The lack of sequence or structural similarity between the terminator proteins of E. coli and B. subtilis and the way in which they recognize their cognate DNA binding sites suggests the two systems have evolved independently of one another (Wake and King, 1997). While closely related species and plasmids harboured by these two organisms also contain homologous replication pause sites in each case 
1 (Griffiths et al., 1998; Hidaka et al., 1988; Meijer et al., 1996; Roecklein et al., 1991), their lack of sequence conservation across diverse bacteria means that it is still unknown how universal the existence of such terminus-region pause sites is across the bacterial domain. However, their apparent independent evolution in the Gram-positive B. subtilis and Gram-negative E. coli suggests that fork traps or pause sites have significant roles in regulating circular chromosome replication and may therefore be expected to be seen in other bacteria.

\section{Mechanisms of DNA replication fork arrest}

\section{Tus: the replication terminator protein of $E$. coli}

Stopping a highly complex replisome that moves with speeds between 650 and $1000 \mathrm{bp} \mathrm{s}^{-1}$ is no mean feat. In both yeast and human cells Tus-Ter complexes were successfully used to block DNA replication (Larsen et al., 2014; Willis et al., 2014). Tus is a monomeric protein of $36 \mathrm{kDa}$ binds that very tightly to its cognate terminators; the Tus-TerB dissociation constant $\left(K_{\mathrm{D}}\right)$ was measured at $3.4 \times 10^{-13} \mathrm{~mol}^{-\mathrm{L}^{-1}}$ and the complex ha a half-life of 550 min under the conditions studied (Hill et al., 1989). Despite the very tight binding, a single Tus-Ter complex is not sufficient to cause significant arrest DNA replication in eukaryotic cells, and between 3 and 7 tandem copies of Ter were used to observe arrest (Larsen et al., 2014; Willis et al., 2014). Furthermore, the E. coli Tus-Ter complex operating in B. subtilis is only $1-2 \%$ efficient in fork arrest compared to arrest of its native E. coli replisome (Andersen et al., 2000). Although it is possible that the binding affinity of Tus-Ter is weaker in the non-native cell environments, these observations may indicate that specificity features other than the Tus-Ter binding affinity could contribute towards stopping a replisome. Also, high-affinity binding of Tus-Ter alone cannot explain the polar (unidirectional) fork-arrest activity observed.

The three-dimensional structure of a Tus-Ter complex, determined using X-ray crystallography (Kamada et al., 1996a), provided clues towards understanding how the complex elicits polar fork-arrest activity. Tus is organized into a large $\mathrm{N}$-terminal domain and a smaller $\mathrm{C}$-terminal domain (Figure 2). Two very long $\beta$ strands join these domains, and are responsible for recognition and tight binding of the Ter sequence, through extensive DNA major-groove contacts. Tus holds the DNA in a girth-like manner so that the DNA is flanked and somewhat enveloped by the two large domains. It is clear from the crystal structure that the replisome would encounter a very different surface of the Tus-Ter complex depending on the direction in which it was travelling - but how does this cause polar fork arrest? It was suggested that the bulky $\alpha$-helical domains at the blocking end of the complex could sterically protect the specific Tus-Ter interactions that are clustered towards the permissive end, thereby sterically preventing the replisome from disrupting the Tus-Ter complex and passing through. From the other direction, the asymmetric protein-DNA contacts are exposed to disruption by the unwinding activity of the ring-shaped DnaB helicase encircling the lagging strand template the at the forefront of the 
replisome. This model thus suggested that Tus-Ter acts as a directional 'molecular clamp' (Kamada et al., 1996a; Wake, 1996). This relatively non-specific mechanism could account for the observations that very different DNA unwinding and sliding proteins (other than the replisome) are arrested in a polar manner by Tus-Ter in vitro, depending on the strand and directionality of protein translocation (Bedrosian and Bastia, 1991; Hidaka et al., 1992; Lee and Kornberg, 1992; Lee et al., 1989; Pandey et al., 2015).

Are there any other mechanisms that contribute to fork arrest and help to arrest the full E. coli replisome in vivo, accounting for the functional specificity noted above? It has been proposed that specific binding between the blocking side of Tus-Ter and the DnaB helicase could inhibit DNA unwinding and underpin the mechanism of fork arrest (Bastia et al., 2008; Mulugu et al., 2001). Point mutations of Tus have been discovered that reduce the effectiveness of fork arrest by Tus but leave its DNA-binding affinity apparently unaffected (Henderson et al., 2001). The existence of such mutations supports the idea that additional important interactions occur when the replisome approaches Tus-Ter. Interestingly, however, these interactions were found to occur between the forked DNA and Tus, rather than between the replisome proteins and Tus, as explained below.

\section{$<$ FIGURE 2 NEAR HERE $>$}

As the replisome approaches Tus-Ter and unwinds DNA up to the blocking end, it was discovered that a conserved cytosine residue (GC(6)) near the blocking face, upon becoming single stranded, swivels and binds a cytosine-binding pocket in Tus and greatly stabilizes the resulting Tus-Ter forked complex (Figure 2a) (Mulcair et al., 2006). This locking effect was termed the "mousetrap" mechanism. In contrast, strand separation if forks are approaching the permissive face resulted in Tus-Ter destabilization (Berghuis et al., 2015; Mulcair et al., 2006). A further study identified that mutations to the GC(6) residue of TerB affected the duration and efficiency of the fork arrest on the T7 helicasepolymerase complex (Pandey et al., 2015). Furthermore, the replisomes that stopped/paused were those that were travelling more slowly, whilst the faster ones were more likely to progress through the Tus/Ter site, since fast replisomes can move through to the $\mathrm{C} 6$ base before $\mathrm{C} 6$ locks onto Tus, thus increasing the probability of replisome displacing Tus and passing through (Elshenawy et al., 2015; Pandey et al., 2015). Consistent with this, a DNA substrate with mismatched base-pairing around the cytosine region that should favour the formation of the lock, blocked more efficiently and could block the faster travelling replisomes as well (Elshenawy et al., 2015; Pandey et al., 2015) .

\section{RTP: the replication terminator protein of $B$. subtilis}

RTP is a winged-helix DNA-binding protein that form a dimer in solution at concentrations above $\sim 10^{-}$

${ }^{6} \mathrm{M}$, and shows no sequence or structural similarity to Tus (Figure 2) (Bussiere et al., 1995; Kralicek et al., 1993; Pai et al., 1996). Each monomer contains four $\alpha$ helices and three $\beta$ strands, with dimerization 
occurring via an antiparallel coiled-coil interaction, giving RTP its symmetrical dimer quaternary structure. Four RTP monomers bind to each Ter sequence to generated the functional complex, which is considered to have two half-sites (one for each of two dimers) designated the A site and the B site (Lewis et al., 1990). The A site binds an RTP dimer much more weakly than the B site, and it is the B site end of the complex from which the replisome can be arrested (Smith and Wake, 1992). The RTPbound B site alone cannot cause arrest, and it has been demonstrated that the highly cooperative binding by RTP, which greatly enhances the affinity of each RTP dimer in the complex, is necessary for fork arrest activity when forks approach the B site (Smith et al., 1996). Interestingly, RTP mostly contacts one the two strands over the A site in Ter DNA (Langley et al., 1993), i.e. the leading strand as a replication fork approaches. The helicase is expected to encircle and translocate along the lagging strand, suggesting that the strand asymmetry might be important in the ability of the replisome to disrupt the complex to proceed when approaching the A-site end, analogous to the mechanism suggested for asymmetric DNA strand contacts by Tus.

The structures of the RTP dimer in complex with B-site DNA sequences revealed the mode of DNA binding (Vivian et al., 2007; Wilce et al., 2001). The primary sequence-specific contacts are made by the N-terminal halves of the two $\alpha 3$ helices of the RTP dimer, which contact two successive major grooves on the same face of the DNA. The positioning of the RTP dimer on the DNA and the knowledge of the centre of symmetry within the two dimer-binding sites has allowed a model of the complete RTPTer complex to be generated (Smith et al., 1996; Wilce et al., 2001). The model suggests that direct contact between the two adjacent RTP dimers bound at the overlapping A and B sites can occur while they are bound to approximately opposite sides of the DNA double helix, potentially explaining the highly cooperative binding observed in full-length terminator complexes. A structure of the complete functional RTP-Ter complex has not yet been obtained; this would greatly assist explanation of the mechanism by which polar replication fork arrest is achieved. Nevertheless, it is considered that the cooperative interaction between RTP dimers confers activity to the B-site end of the whole RTP-Ter complex, and the differential binding affinity and strand-asymmetry differences of RTP bound to the A and B sites, are important in establishing the functional polarity of the RTP-Ter complex (Duggin et al., 1999; Kralicek et al., 1997; Langley et al., 1993; Smith et al., 1996). While there was generally a correlation between RTP-Ter binding affinity and fork arrest efficiency, some mutant Ter sites do not strictly conform to this correlation, suggesting that the conformation of RTP-Ter has a meaningful role in the mechanism of fork arrest (Duggin et al., 2005). It was further suggested that the chock-like shape of RTP on the DNA could assist in wedging the advancing replisome. As a test of this hypothesis, peptides of differing size were attached to the C-terminus of RTP, placing them away from the DNAbinding site and in a position that was expected to come into contact with the advancing replisome (see Figure 2b) (Duggin, 2006). These RTP fusions had unaltered Ter DNA binding affinity, but showed fork arrest activity that reduced with increasing size of the attached peptide. This suggested that the replisome could remove the larger RTP-peptide fusions more easily from Ter DNA, and was taken to 
be most consistent with the molecular chock model. Substantive evidence for specific interactions between RTP and the replisome is still lacking, since RTP point mutants examined so far had normal function or are defective in DNA binding (Duggin et al., 1999; Griffiths et al., 1998; Manna et al., 1996). Future studies should gain an understanding of the structure and dynamics of the whole RTP complex with forked DNA and B. subtilis replisome components.

\section{Fork arrest at other sites}

Fork arrest in B. subtilis also occurs at sites within $200 \mathrm{~kb}$ of oriC under conditions that induce the stringent response. The delay to replication under these nutrient-deprived conditions acts as a type of replication checkpoint, concurrent with other metabolic changes associated with the stringent response. The nature of the B. subtilis replication checkpoint differs markedly from E. coli, in which initiation of DNA replication at oriC is inhibited during the stringent response (Schreiber et al., 1995). In B. subtilis, possibly three of the so-called STer sites exist to the left of oriC and two to the right. The second STer site to the left of oriC, named LSTer2, has been partially characterized (Autret et al., 1999). It was found that both RTP and the stringent response regulator protein RelA were required in vivo for fork-arrest activity at a segment identified to contain LSTer2. Also, the activation of fork blocking at LSTer2 depended on induction of stringent response conditions (Autret et al., 1999; Gautam and Bastia, 2001). Upon examination, the chromosomal segment containing LSTer2 was found to contain a sequence exhibiting similarity to the consensus B site of the terminus-region terminators. Significantly, specific deletion of the B-like site abolished stringent fork-arrest activity on the cloned fragment containing LSTer2 (Autret et al., 1999). The B-like sequence was found to bind purified RTP in vitro, but only one RTP dimer bound efficiently to the DNA fragments examined (Autret et al., 1999; Gautam and Bastia, 2001). Although these findings suggest an alternative make-up of the stringent and terminus-region terminators, it remains to be seen what factors are directly responsible for the regulation of stringent termination.

Fork arrest can also occur as an "unplanned" event, caused by collision of the replisome with other DNA-binding proteins or by encounter of DNA damage on the template. These tend to be stochastic events that could potentially take place at any position on the chromosome. In E. coli it has been shown that the major cause of replisome stalling is encounter with proteins bound to the template DNA, and of these RNA polymerase is the major culprit (Gupta et al., 2013). The accessory helicase Rep is travelling with replisomes and aids in displacing proteins if a replisome gets blocked (Guy et al., 2009). In the absence of Rep, UvrD may aid in this process, or may be required to resolve recombination intermediates that result from replisome collapse (Gupta et al., 2013; Petit and Ehrlich, 2002). PcrA likely plays a similar role in Gram positive bacteria (Naqvi et al., 2003). Genomes of bacteria have evolved to favour co-directionality of transcription with replication to avoid the potentially damaging head-on collisions that could otherwise occur (Rudolph et al., 2007). 
Proteins bound to DNA have been utilised to artificially block DNA replication forks and are useful tools for examining chromosome biology; these range from introduction of ectopic Ter sites to addition of arrays of transcription repressor binding sites into the chromosome (Bidnenko et al., 2002; Payne et al., 2006; Possoz et al., 2006). When an ectopic highly efficient Ter site was placed roughly halfway round one of the chromosome arms to prevent replication proceeding further toward the terminus, cell survival was found to be dependent on RecA (Bidnenko et al., 2002). It was, therefore, proposed that a second fork from oriC collides with the stalled fork from behind and produces a double strand break that is repaired by homologous recombination. This repair also displaces Tus-Ter allowing replication to proceed past this point, although the precise mechanism for this is unknown. Fluorescently tagged transcriptional repressor proteins have been widely used to bind to arrays of their cognate binding sites to mark specific chromosomal loci to study chromosome dynamics in many organisms (Lau et al., 2003; Matzke et al., 2005; Straight et al., 1996). With a sufficiently large array of protein-DNA sites in tandem repeat, overproduction of the repressor protein leads to a replication roadblock that cannot be overcome (Possoz et al., 2006). Replication forks blocked at these repressor arrays have a half-life of 3-5 minutes, indicating that any long-lived unresolved protein block is a potential site for replisome dissociation (Mettrick and Grainge, 2016).

Another artificial situation is the overproduction of Tus. This activates additional weaker matches to the Ter site consensus in the E. coli chromosome, some of which are within reading frames and are considered unlikely to have evolved specifically as Ter sites (Duggin and Bell, 2009). Some of these sites are orientated in the blocking orientation of oriC-initiated replication and show a detectable pause in sensitive 2D gel electrophoresis assays when Tus is overproduced; these sites are not functional at physiological levels of Tus and were termed pseudo-terminators or $p$ Ter sites. By this definition, some of the outermost Ter sites previously assigned as part of replication fork trap due to their orientation in the genome would be considered $p T e r$ sites. The overproduction of Tus also caused some of the stronger chromosomal Ter sites, such as $\operatorname{Ter} G$, to show detectable pausing of replication forks approaching the normally permissive end of the complex (Duggin and Bell, 2009). It was considered that this might reflect an initial very brief pause by the replisome at the permissive end of Tus-Ter, that is normally undetectable in 2D gel assays, but with overproduced Tus, the Ter site can be re-bound by Tus at a rate that successfully competes for the DNA with the replisome, resulting in a weakly detectable pause.

\section{Resolution of Daughter Chromosomes}

After the fusion of two replication forks, some post-replicative events are frequently required so that complete separation of daughter chromosomes can be achieved. These events are the untangling of topologically interlinked daughter chromosomes (decatenation) and the resolution of dimeric chromosomes to monomeric daughter chromosomes that each newly forming cell eventually inherits. 
During DNA replication the positive supercoiling that builds up as the replication fork progresses must be continuously removed. The primary enzyme involved in removing such superhelical stress is DNA gyrase, a type II topoisomerase (Kreuzer and Cozzarelli, 1979; Peebles et al., 1979). Despite the action of DNA gyrase, some so-called precatenation of the sister duplexes occurs as replication forks progress. The positive superhelical stress ahead of the fork can force the fork and associated proteins to rotate, thereby interwrapping the two daughter chromosomes. If not removed the precatentation causes the two chromosomes to remain topologically linked (catenated) after termination of DNA replication (ReyesLamothe et al., 2012). This barrier to chromosome segregation is largely solved by another type II topoisomerase, topoisomerase IV, which specifically resolves these catenanes (Espeli et al., 2003; Hojgaard et al., 1999; Reyes-Lamothe et al., 2012). This enzyme transiently cuts both strands of one daughter chromosome and then passes DNA from the other through the resulting gap before rejoining, thus removing an interlinkage.

The other obstacle to the separation of the two daughter chromosomes is a chromosome dimer- the result of an odd number of homologous recombination events between the two duplicated portions of the replicating chromosome. Homologous recombination is a significant by-product of the reactions needed to restart any replication fork that has stalled undesirably during DNA replication (Cox, 2001). This can eventually lead to the formation of a chromosome dimer. Both the E. coli and B. subtilis chromosomes contain a special recombination site, located within the terminus region, which allows the resolution of the chromosome dimers that might have formed; in E. coli it is close to the innermost Ter site TerC, while in B. subtilis it is adjacent to TerVIII. The site is named dif (deletion-induced filamentation) because its deletion leads to a filamentation phenotype as a result of the cell's inability to partition the joined chromosomes and complete cell division (the rod-shaped cells continue to grow lengthwise and form long filaments that eventually die) (Kuempel et al., 1991). The two copies of dif present in a chromosome dimer are juxtaposed during the late stages of chromosome segregation, which allows DNA strand exchange and chromosome dimer resolution into monomers (Barre et al., 2001; Reyes-Lamothe et al., 2012).

Several factors are required for functioning of the dif site. dif is composed of a 28-bp core sequence that acts as a binding site for the site-specific recombinases XerC and XerD (named CodV and RipX respectively in $B$. subtilis). In E. coli, chromosome dimer resolution at dif also requires the membraneanchored FtsK, a large multifunctional protein (Grainge, 2013). FtsK is anchored at the division septum and loads onto polar sequences called KOPS (FtsK orienting polar sequences). These sequences are oriented towards the dif resolution site, which allows FtsK to distribute chromosomal DNA to either side of the septum, thereby preventing chromosome 'guillotining' (Bigot et al., 2005). Translocation of FtsK eventually will bring the dif site to the septal region (Barre et al., 2001). FtsK acts on the XerCDdif complex inside the cell during the concomitant dimer resolution and cell division events. It directs the sequential activity of XerD, then of XerC, which imparts directionality to the recombination event 
to resolve chromosome dimers to monomers effectively (and prevent formation of a dimer from two monomers) (Aussel et al., 2002; Barre et al., 2001; Grainge et al., 2011). In B. subtilis, there are two FtsK homologues; the DNA motor proteins SpoIIIE and SftA appear to work synergistically in clearing chromosomal DNA from the site of septation and in chromosome dimer resolution, although the mechanistic details remain less well defined than in E. coli (Biller and Burkholder, 2009; Kaimer et al., 2009; Kaimer et al., 2011).

Archaea with circular chromosomes are also expected to generate interlinked and dimer chromosomes that require resolution. While archaea may not contain specific fork traps (or are yet to be discovered), they contain Xer-dif dimer resolution systems homologous to bacteria (Cortez et al., 2010; Duggin et al., 2011). Some of the dif sites of archaea are not located in the expected terminus region, and this may reflect a very different mode of chromosome segregation and division control in these archaea compared to bacteria.

\section{Physiological Roles of the Replication Fork Trap}

The defined architecture of the E. coli chromosome with a single replication origin and a fork trap directly opposite dictates that the majority of replication fork fusion events will take place within the innermost Ter sites, even if one fork was to be delayed by obstacles such as protein-DNA complexes, DNA secondary structures or DNA lesions (cf Figures 1a \& 4a). In E. coli, TerC, one of the innermost Ter sites, is located almost directly opposite oriC, while the second innermost Ter site, TerA, is $270 \mathrm{~kb}$ away. However, replication forks do not meet systematically at Ter/Tus complexes, as shown by early labelling experiments (Bouche et al., 1982) and more recently by high-resolution replication profiles, which allow quantification of the relative copy number of chromosomal areas. The majority of forks fuse in wild type $E$. coli cells close to the arithmetic mid-point, and the same region where fork fusions is observed both in the presence and absence of a functional fork trap (Dimude et al., 2016; Ivanova et al., 2015; Rudolph et al., 2013), suggesting that both forks normally move with similar speeds and, therefore, fuse freely within the region of the innermost Ter sites. The fork trap will come into play if one of the two forks is delayed on its way to the terminus, and 2D DNA gel electrophoresis, which can visualise replication intermediates such as forks paused at a Ter/Tus complex, showed that in normally growing wild type E. coli cells forks indeed arrest occasionally at Ter/Tus complexes. The strongest signal was observed at $\operatorname{Ter} C$, as might be expected given its proximity to the natural fusion site, followed by TerA (Duggin and Bell, 2009; Maduike et al., 2014).

What might be the physiological role of the replication fork trap? It has been suggested that it maintains the co-directionality of transcription and replication in the terminus. Both in B. subtilis and E. coli the vast majority of highly transcribed genes, such as $r r n$ operons encoding ribosomal RNA, as well as genes encoding ribosomal proteins, are transcribed co-directionally with the direction of replication 
(Figure 1) (Brewer, 1988; McLean et al., 1998). Indeed, there is ample evidence that head-on collisions of replication and transcription complexes pose a significant challenge to cells (Kim and JinksRobertson, 2012; McGlynn et al., 2012; Merrikh et al., 2012; Rudolph et al., 2007), especially if they occur at highly-transcribed genes such as rrn operons (Boubakri et al., 2010; De Septenville et al., 2012; Ivanova et al., 2015; Srivatsan et al., 2010; Wang et al., 2007). The fork trap would prevent clashes between replication and transcription by ensuring that replication forks from one replichore are not allowed to proceed into the opposite replichore (Brewer, 1988; Rudolph et al., 2007).

However, the situation is complex. While in E. coli over $90 \%$ of highly transcribed genes replication and transcription proceed co-directionally, genome-wide co-directionality is only just above average (55\%) (Brewer, 1988; McLean et al., 1998). All rrn operons and the vast majority of genes encoding for ribosomal proteins are located in the origin-proximal half of the chromosome (Dimude et al., 2016; Jin et al., 2012). Thus, forks escaping the termination area would have to replicate at least another $1 / 4$ of the chromosome (1 Mbp) before reaching any of these highly-transcribed genes, which would only happen if the other fork is arrested for a considerable period. tRNA genes, also highly transcribed under fast growth conditions and more globally distributed throughout the chromosome, are mostly oriented co-directional in the origin-proximal half of the chromosome. Surprisingly, in the origin-distal half, orientation is much more heterogeneous, with a mild bias towards the head-on orientation for replication coming from oriC (Dimude et al., 2016). Thus, replication escaping the termination area might in fact encounter fewer problems than forks coming from oriC. There is little doubt that head-on collisions between replication and transcription can be very problematic for cells, but given the chromosome organisation it is debatable whether avoiding clashes between replication and highly transcribed areas are the main purpose of the terminus region in E. coli. On the other hand, B. subtilis shows a stronger co-directionality of replication and transcription.

Replication proceeding into the opposite replichore can be studied in cells containing ectopic replication origins in addition to oriC. In cells that carry an ectopic replication origin called oriZ (Figure 4b), located at the lac $Z$ region, roughly $1 / 2$ way round the clockwise replichore, replication of the chromosome becomes asymmetric (Ivanova et al., 2015; Wang et al., 2011). Replication initiating at oriC and proceeding counter-clockwise will have to replicate $1 / 2$ of the chromosome, while forks initiated at oriZ and proceeding clockwise will get blocked at Ter/Tus complexes after replicating only $1 / 4$ of the chromosome (Figure $4 \mathrm{~b}$, green arrows). Upon deletion of tus the forks from oriZ will be able to proceed into the opposite replichore in an orientation opposite to normal (Figure $4 \mathrm{c}$, blue arrows). If forks travelling in an orientation opposite to normal were delayed due to head-on collisions with transcribing RNA polymerase complexes, the reduced fork speed should shift the fork fusion point away from the arithmetic mid-point (mid-way between oriC and oriZ) towards the termination area (Figure $4 \mathrm{c}$, grey arrows). Similar experiments in $\Delta t u s$ cells with an ectopic origin called oriX in the opposite replichore showed a fork fusion point essentially located at the arithmetic mid-point between oriC and 
ori $X$, confirming that the speed of forks coming from oriC and forks escaping the termination area is at least similar (Dimude et al., 2018). Thus forks escaping the termination area appear to proceed with few problems, supporting the idea that avoiding replication-transcription clashes is unlikely to be the main purpose of the termination area.

A termination area forming an absolute fork trap actually poses a serious threat to cells; if one fork is very delayed at an obstacle, replication cannot be completed by the opposite fork because it will get blocked in the termination area (Figure 4a). Any stalled fork will have to be restarted or the cell affected will be in danger of dying, explaining the prominent role of replication restart proteins such as PriA in bacteria, which aid the recruitment of replication fork components at sites of damage (Gabbai and Marians, 2010). This scenario can be simulated in cells that carry the ectopic replication origin oriZ but in which oriC is deleted. If the fork trap is active then $3 / 4$ of the chromosome has to be replicated by the fork proceeding counter-clockwise, as the opposite fork will be blocked by Ter/Tus complexes after replicating $1 / 4$ of the chromosome (Figure $4 \mathrm{~d}$ i, green arrows). The doubling time of this construct was shown to be delayed to about twice that of normal wild type cells (40 vs $20 \mathrm{~min}$ ) (Ivanova et al., 2015). The inactivation of the replication fork trap by deleting the tus gene partially suppressed the slow growth phenotype of $\triangle$ oriC oriZ cells (doubling time: $29 \mathrm{~min}$ ), demonstrating that the replication fork trap indeed inhibits fast growth if one fork is stalled at an obstacle for a long period of time (Figure 4dii) (Dimude et al., 2016; Ivanova et al., 2015).

Are there other phenotypes associated with the replication fork trap that might shed light on its most important physiological role? Various experimental approaches have shown that over-replication can occur at fork fusion sites. For example, plasmid R1 is replicated by a single replication fork moving uni-directionally around the plasmid until it is arrested at a single Ter/Tus complex (Nordstrom, 2006). In the absence of Tus protein an accumulation of complex branched DNA structures, plasmid multimers and rolling circle replication intermediates was observed (Krabbe et al., 1997). It was suggested that the helicase of the replication fork, upon reaching the already replicated area, might displace the existing nascent ends, which will generate intermediates that can serve as substrates for the continuation of replication (Krabbe et al., 1997). Similarly, in vitro studies had shown before that Tus prevented overreplication of a circular DNA containing a fork trap in vitro (Hiasa and Marians, 1994), lending support to a possible function of the bacterial replication fork trap in preventing over-replication.

A low level of chromosomal over-replication was also observed in the absence of Tus in otherwise wild type $E$. coli cells. This over-replication was exacerbated if additional mutations were added, such as point mutants in the polA gene, which encodes for polymerase I, a polymerase involved in DNA damage repair and Okazaki fragment maturation (Kurth and O'Donnell, 2009), suggesting one of the roles of Pol I might be to help bring DNA replication to a successful conclusion in the terminus region (Markovitz, 2005). Similarly, the absence of RTP in B. subtilis leads to phenotypes suggesting that an 
increased number of chromosomal dimers are formed, which might also be the result of problems with terminating DNA replication accurately (Duggin et al., 2008; Lemon et al., 2001).

An even stronger effect of a lack of Tus protein was found more recently in cells lacking RecG helicase. The analysis of replication profiles in $\Delta \operatorname{rec} G$ cells demonstrated a peak of over-replication in the termination area (Figure 5a) (Dimude et al., 2015; Rudolph et al., 2013; Wendel et al., 2014). Inactivation of the replication fork trap by deletion of tus allowed the observed over-replication to extend beyond the termination area in both directions. The forks which progress in a direction opposite to normal will suffer from head-on collisions with transcribing RNA polymerase complexes, especially at highly transcribed $r r n$ operons, as described above. This can be alleviated by a point mutation called rpo*, which destabilises ternary RNA polymerase complexes (Rudolph et al., 2007; Trautinger et al., 2005). $\Delta r e c G \Delta t u s ~ r p o *$ cells can survive the deletion of the entire oriC (Figure 5b), and appear to replicate by multiple rounds of recombination-based over-replication. Survival depends critically on the absence of Tus protein and is one of the strongest phenotypes of $\Delta t u s$ cells reported so far (Dimude et al., 2015; Rudolph et al., 2013). $\Delta r e c G \Delta t u s ~ r p o *$ cells in which oriC firing was inhibited showed an inverted replication profile where no sign of initiation is seen in the oriC area but, rather paradoxically, the highest levels of synthesis are observed in the termination area where forks normally fuse (Figure 5c) (Dimude et al., 2015; Rudolph et al., 2013). Thus, the level of over-replication in the absence of RecG is so substantial that it can sustain growth and division in the absence of oriC firing as long as the fork trap area is inactivated by deletion of tus and head-on replication-transcription conflicts are alleviated by an rpo* point mutation.

What might be the reason for over-replication specifically in the termination area? One event that is specific for the termination area is the fusion of two replication forks (Figure 6a). We are only beginning to understand the events associated with two fusing forks, but the analysis of the over-replication seen in $\Delta r e c G$ cells suggests that it might originate from DNA intermediates similar to those proposed for R1 replication (Dimude et al., 2016; Dimude et al., 2015; Krabbe et al., 1997; Lloyd and Rudolph, 2016; Rudolph et al., 2013). Upon fusion of two replication forks the helicase of one fork could displace leading strand of the opposing fork, resulting in the formation of a 3' ssDNA flap structure (Figure 3b). 3' flaps are an excellent substrate for RecG helicase (Bianco, 2015; McGlynn et al., 2001; Tanaka and Masai, 2006) and, in its presence, would be rapidly processed. 3' flaps also could be degraded by 3' single-stranded exonucleases, an idea supported by the observation that cells lacking all major 3' exonucleases also show over-replication in the terminus area (Rudolph et al., 2010a; Wendel et al., 2014). In the absence of either RecG or 3' exonucleases a 3' flap is likely to persist for longer and can be processed by the main replication fork restart protein PriA, which will trigger the assembly of a new replication fork (Figure 6c). Progression of such a fork will result in the generation of a double-stranded DNA end. Any such DNA end will be rapidly processed by the homologous recombination proteins RecBCD and RecA, leading to a strand invasion (a so-called D-loop) (Dimude et al., 2016; Dimude et 
al., 2015; Rudolph et al., 2010a; Rudolph et al., 2010b; Rudolph et al., 2009; Rudolph et al., 2013). A D-loop is another substrate for PriA, which might establish another replication fork that would move in the opposite direction (Figure 6d). Progression of newly established forks will normally be blocked by $\mathrm{Ter} /$ Tus complexes as they proceed towards oriC, explaining why over-replication in cells lacking RecG is tightly restricted to the termination area. A recent analysis shows that over-replication in the absence of RecG can also be triggered outside of the termination area if forks are forced to fuse in an ectopic location (Midgley-Smith et al., 2018), strongly supporting the idea that the over-replication observed is triggered by events associated with the fusion of two replication forks.

These data suggest that one role of the termination area might be to contain the over-replication triggered by replication forks fusions to a defined area where appropriate processing can take place. The fact that increased levels of over-replication in the termination area are observed in cells lacking a number of proteins such as RecG, 3' exonucleases, DNA polymerase I and RecD (Markovitz, 2005; Rudolph et al., 2013; Wendel et al., 2014) suggests that a surprising number of proteins might be involved in the processing of fork fusion intermediates. In addition, processing of fork fusion intermediates by homologous recombination proteins will increase the frequency of recombination events in the termination area. Both over-replication of already replicated DNA and increased recombination frequencies have been found to contribute to genomic instability (Alexander and OrrWeaver, 2016; Blow and Gillespie, 2008; Finkel et al., 2007; Tomasetti et al., 2017), providing another explanation for the importance of the replication fork trap.

That the processing of such intermediates might be of vital importance is illustrated by the fact that the consequences in cells lacking both RecG and 3' exonucleases are so severe that they are inviable (Rudolph et al., 2010a). A similar lethality was observed for the combination of a polA mutation with a $r e c G$ deletion (Hong et al., 1995; Upton et al., 2014; Zhang et al., 2010). In addition, in $\Delta r e c G$ cells carrying the ectopic replication origin oriZ the over-replication in the termination area was vastly exacerbated (Figure 5d). Over-replication is still observed in $\triangle \operatorname{rec} G$ cells lacking Tus terminator protein, highlighting that over-replication is not triggered at forks arrested at Ter/Tus complexes. However, both in vitro and in vivo data suggest that arrested forks have a limited half-life and are disassembled after a relatively short period of time (Marians et al., 1998; McGlynn and Guy, 2008; Mettrick and Grainge, 2016). Thus, in $\Delta r e c G$ cells in which replication forks are stalled at Ter/Tus complexes for an extended period of time, at least some forks will be disassembled. A collision event between a replisome and a partially disassembled fork might be particularly prone for triggering over-replication. In contrast, this risk will be considerably lower if one replisome is paused via the arrest of the helicase at a Ter/Tus complex, as both Tus and the arrested helicase will act as a "buffer" that stops progression of the helicase of the opposing fork. In wild type cells the stable arrest of replisomes at Ter/Tus complexes for some time might therefore be an important feature: it will actively prevent processing of stalled 
forks while the second fork is likely to reach the termination area soon after (Midgley-Smith et al., 2018)

In line with the idea that the termination area might contain potentially harmful intermediates it was impossible to delete oriC in $\operatorname{oriZ}^{+} \Delta \mathrm{rec} G$ cells. However, oriZ $Z^{+} \Delta$ oriC $\Delta r e c G$ was generated without much difficulty if the replication fork trap was inactivated by a tus deletion, confirming that in cells which replicate their chromosome asymmetrically from an ectopic origin things go catastrophically wrong, specifically in the termination area, in the absence of RecG (Midgley-Smith et al., 2018).

The comparison of the replication fork trap components in E. coli and B. subtilis have revealed no significant sequence or structural similarity, indicating that both systems might have evolved independently (Neylon et al., 2005), suggesting that a fork trap has a very important physiological function. But, so far the reported phenotypes for $\Delta t u s$ and $\Delta r t p$ single mutants are rather mild. The hypothesis that the fusion of two replication forks, a necessary part of chromosome duplication, can have harmful consequences might offer not only an explanation for the importance of a fork trap, but might also at least in part explain why bacterial chromosomes are with almost no exception replicated from a single replication origin (Gao, 2015; Gao and Zhang, 2008). A chromosomal architecture with a single replication origin and a fork trap area, which per se defines two replichores, allows not only the co-orientation of replication and highly transcribed genes, but also minimises the number of replication fork fusion events to exactly one per cell cycle. It is tempting to speculate that the replication fork trap, rather than defining the location of fork fusion events, has evolved around the location where forks fuse naturally, and that over-replication can be safely contained in this region if the normal processing by proteins such as RecG, Pol I or 3' exonucleases fails for some reason.

\section{Glossary}

\section{Circular chromosome}

The double-stranded bacterial DNA (deoxyribonucleic acid) chromosome forms one complete circle with no free ends.

\section{Fork arrest}

\section{Helicase}

Arrest (or pausing) of a replication fork at a defined location on the chromosome.

\section{Replication fork}

An enzyme that unwinds duplex DNA in an ATP-dependent manner.

Junctions in partially replicated DNA where the newly synthesized daughter strands meet the unreplicated parental DNA.

\section{Replication fork trap}

Two groups of terminators with opposite orientations arranged to trap replication forks

\section{Replicon} in the terminus region of the chromosome.

\section{Replisome}

A region of DNA replicated from a single origin of replication. It can be either a whole DNA molecule or part of one.

$$
-16-
$$


A large multiprotein complex, located at the apex of the replication fork, that catalyses DNA replication.

Terminator

A DNA site that, upon binding a terminator protein, can bring about arrest (or pausing) of a replication fork.

Terminator protein

A protein that, upon binding to a specific DNA site known as a terminator, causes arrest (or pausing) of a replication fork.

\section{Terminus region}

A restricted and defined segment of a bacterial chromosome in which replication forks meet and fuse. 
Ahn, K.S., M.S. Malo, M.T. Smith, and R.G. Wake. 1993. Autoregulation of the gene encoding the replication terminator protein of Bacillus subtilis. Gene. 132:7-13.

Alexander, J.L., and T.L. Orr-Weaver. 2016. Replication fork instability and the consequences of fork collisions from rereplication. Genes Dev. 30:2241-2252.

Andersen, P.A., A.A. Griffiths, I.G. Duggin, and R.G. Wake. 2000. Functional specificity of the replication fork-arrest complexes of Bacillus subtilis and Escherichia coli: significant specificity for Tus-Ter functioning in E. coli. Mol Microbiol. 36:13271335.

Aussel, L., F.X. Barre, M. Aroyo, A. Stasiak, A.Z. Stasiak, and D. Sherratt. 2002. FtsK Is a DNA motor protein that activates chromosome dimer resolution by switching the catalytic state of the XerC and XerD recombinases. Cell. 108:195-205.

Autret, S., A. Levine, F. Vannier, Y. Fujita, and S.J. Seror. 1999. The replication checkpoint control in Bacillus subtilis: identification of a novel RTP-binding sequence essential for the replication fork arrest after induction of the stringent response. Mol Microbiol. 31:1665-1679.

Barre, F.X., B. Soballe, B. Michel, M. Aroyo, M. Robertson, and D. Sherratt. 2001. Circles: the replication-recombination-chromosome segregation connection. Proc Natl Acad Sci U S A. 98:8189-8195.

Bastia, D., S. Zzaman, G. Krings, M. Saxena, X. Peng, and M.M. Greenberg. 2008. Replication termination mechanism as revealed by Tus-mediated polar arrest of a sliding helicase. Proc Natl Acad Sci U S A. 105:12831-12836.

Beattie, T.R., and R. Reyes-Lamothe. 2015. A Replisome's journey through the bacterial chromosome. Front Microbiol. 6:562.

Bedrosian, C.L., and D. Bastia. 1991. Escherichia coli replication terminator protein impedes simian virus 40 (SV40) DNA replication fork movement and SV40 large tumor antigen helicase activity in vitro at a prokaryotic terminus sequence. Proc Natl Acad Sci U S A. 88:2618-2622.

Berghuis, B.A., D. Dulin, Z.Q. Xu, T. van Laar, B. Cross, R. Janissen, S. Jergic, N.E. Dixon, M. Depken, and N.H. Dekker. 2015. Strand separation establishes a sustained lock at the Tus-Ter replication fork barrier. Nat Chem Biol. 11:579-585.

Bianco, P.R. 2015. I came to a fork in the DNA and there was RecG. Prog Biophys Mol Biol. 117:166-173.

Bidnenko, V., S.D. Ehrlich, and B. Michel. 2002. Replication fork collapse at replication terminator sequences. EMBO J. 21:3898-3907.

Bigot, S., O.A. Saleh, C. Lesterlin, C. Pages, M. El Karoui, C. Dennis, M. Grigoriev, J.F. Allemand, F.X. Barre, and F. Cornet. 2005. KOPS: DNA motifs that control E. coli chromosome segregation by orienting the FtsK translocase. EMBO J. 24:37703780.

Biller, S.J., and W.F. Burkholder. 2009. The Bacillus subtilis SftA (YtpS) and SpoIIIE DNA translocases play distinct roles in growing cells to ensure faithful chromosome partitioning. Mol Microbiol. 74:790-809.

Blow, J.J., and P.J. Gillespie. 2008. Replication licensing and cancer--a fatal entanglement? Nat Rev Cancer. 8:799-806.

Boubakri, H., A.L. de Septenville, E. Viguera, and B. Michel. 2010. The helicases DinG, Rep and UvrD cooperate to promote replication across transcription units in vivo. EMBO J. 29:145-157.

Bouche, J.P., J.P. Gelugne, J. Louarn, J.M. Louarn, and K. Kaiser. 1982. Relationships between the physical and genetic maps of a $470 \times 10$ (3) base-pair region around the terminus of Escherichia coli K12 DNA replication. J Mol Biol. 154:21-32.

Brewer, B.J. 1988. When polymerases collide: replication and the transcriptional organization of the E. coli chromosome. Cell. 53:679-686. 
Bussiere, D.E., D. Bastia, and S.W. White. 1995. Crystal structure of the replication terminator protein from B. subtilis at 2.6 A. Cell. 80:651-660.

Cortez, D., S. Quevillon-Cheruel, S. Gribaldo, N. Desnoues, G. Sezonov, P. Forterre, and M.C. Serre. 2010. Evidence for a Xer/dif system for chromosome resolution in archaea. PLoS Genet. 6:e1001166.

Coskun-Ari, F.F., and T.M. Hill. 1997. Sequence-specific interactions in the Tus-Ter complex and the effect of base pair substitutions on arrest of DNA replication in Escherichia coli. J Biol Chem. 272:26448-26456.

Cox, M.M. 2001. Historical overview: searching for replication help in all of the rec places. Proc Natl Acad Sci U S A. 98:8173-8180.

De Septenville, A.L., S. Duigou, H. Boubakri, and B. Michel. 2012. Replication fork reversal after replication-transcription collision. PLoS Genet. 8:e1002622.

Dimude, J.U., S.L. Midgley-Smith, M. Stein, and C.J. Rudolph. 2016. Replication Termination: Containing Fork Fusion-Mediated Pathologies in Escherichia coli. Genes (Basel). 7.

Dimude, J.U., M. Stein, E.E. Andrzejewska, M.S. Khalifa, A. Gajdosova, R. Retkute, O. Skovgaard, and C.J. Rudolph. 2018. Origins Left, Right, and Centre: Increasing the Number of Initiation Sites in the Escherichia coli Chromosome. Genes (Basel). 9.

Dimude, J.U., A. Stockum, S.L. Midgley-Smith, A.L. Upton, H.A. Foster, A. Khan, N.J. Saunders, R. Retkute, and C.J. Rudolph. 2015. The Consequences of Replicating in the Wrong Orientation: Bacterial Chromosome Duplication without an Active Replication Origin. MBio. 6:e01294-01215.

Duggin, I.G. 2006. DNA replication fork arrest by the Bacillus subtilis RTP-DNA complex involves a mechanism that is independent of the affinity of RTP-DNA binding. $J$ Mol Biol. 361:1-6.

Duggin, I.G., P.A. Andersen, M.T. Smith, J.A. Wilce, G.F. King, and R.G. Wake. 1999. Site-directed mutants of RTP of Bacillus subtilis and the mechanism of replication fork arrest. J Mol Biol. 286:1325-1335.

Duggin, I.G., and S.D. Bell. 2009. Termination structures in the Escherichia coli chromosome replication fork trap. J Mol Biol. 387:532-539.

Duggin, I.G., N. Dubarry, and S.D. Bell. 2011. Replication termination and chromosome dimer resolution in the archaeon Sulfolobus solfataricus. EMBO J. 30:145-153.

Duggin, I.G., J.M. Matthews, N.E. Dixon, R.G. Wake, and J.P. Mackay. 2005. A complex mechanism determines polarity of DNA replication fork arrest by the replication terminator complex of Bacillus subtilis. J Biol Chem. 280:13105-13113.

Duggin, I.G., R.G. Wake, S.D. Bell, and T.M. Hill. 2008. The replication fork trap and termination of chromosome replication. Mol Microbiol. 70:1323-1333.

Elshenawy, M.M., S. Jergic, Z.Q. Xu, M.A. Sobhy, M. Takahashi, A.J. Oakley, N.E. Dixon, and S.M. Hamdan. 2015. Replisome speed determines the efficiency of the TusTer replication termination barrier. Nature. 525:394-398.

Espeli, O., C. Levine, H. Hassing, and K.J. Marians. 2003. Temporal regulation of topoisomerase IV activity in E. coli. Mol Cell. 11:189-201.

Finkel, T., M. Serrano, and M.A. Blasco. 2007. The common biology of cancer and ageing. Nature. 448:767-774.

Gabbai, C.B., and K.J. Marians. 2010. Recruitment to stalled replication forks of the PriA DNA helicase and replisome-loading activities is essential for survival. DNA Repair (Amst). 9:202-209.

Gambus, A. 2017. Termination of Eukaryotic Replication Forks. Adv Exp Med Biol. 1042:163-187.

Gao, F. 2015. Bacteria may have multiple replication origins. Front Microbiol. 6:324.

Gao, F., and C.T. Zhang. 2008. Ori-Finder: a web-based system for finding oriCs in unannotated bacterial genomes. BMC Bioinformatics. 9:79.

Gautam, A., and D. Bastia. 2001. A replication terminus located at or near a replication checkpoint of Bacillus subtilis functions independently of stringent control. $\mathrm{J} \mathrm{Biol}$ Chem. 276:8771-8777. 
Grainge, I. 2013. Simple topology: FtsK-directed recombination at the dif site. Biochem Soc Trans. 41:595-600.

Grainge, I., C. Lesterlin, and D.J. Sherratt. 2011. Activation of XerCD-dif recombination by the FtsK DNA translocase. Nucleic Acids Res. 39:5140-5148.

Griffiths, A.A., P.A. Andersen, and R.G. Wake. 1998. Replication terminator proteinbased replication fork-arrest systems in various Bacillus species. J Bacteriol. 180:3360-3367.

Griffiths, A.A., and R.G. Wake. 2000. Utilization of subsidiary chromosomal replication terminators in Bacillus subtilis. J Bacteriol. 182:1448-1451.

Gupta, M.K., C.P. Guy, J.T. Yeeles, J. Atkinson, H. Bell, R.G. Lloyd, K.J. Marians, and P. McGlynn. 2013. Protein-DNA complexes are the primary sources of replication fork pausing in Escherichia coli. Proc Natl Acad Sci U S A. 110:7252-7257.

Guy, C.P., J. Atkinson, M.K. Gupta, A.A. Mahdi, E.J. Gwynn, C.J. Rudolph, P.B. Moon, I.C. van Knippenberg, C.J. Cadman, M.S. Dillingham, R.G. Lloyd, and P. McGlynn. 2009. Rep provides a second motor at the replisome to promote duplication of protein-bound DNA. Mol Cell. 36:654-666.

Hawkins, M., S. Malla, M.J. Blythe, C.A. Nieduszynski, and T. Allers. 2013. Accelerated growth in the absence of DNA replication origins. Nature. 503:544-547.

Helmstetter, C., S. Cooper, O. Pierucci, and E. Revelas. 1968. On the bacterial life sequence. Cold Spring Harb Symp Quant Biol. 33:809-822.

Henderson, T.A., A.F. Nilles, M. Valjavec-Gratian, and T.M. Hill. 2001. Site-directed mutagenesis and phylogenetic comparisons of the Escherichia coli Tus protein: DNA-protein interactions alone can not account for Tus activity. Mol Genet Genomics. 265:941-953.

Hiasa, H., and K.J. Marians. 1994. Tus prevents overreplication of oriC plasmid DNA. J Biol Chem. 269:26959-26968.

Hidaka, M., M. Akiyama, and T. Horiuchi. 1988. A consensus sequence of three DNA replication terminus sites on the $\mathrm{E}$. coli chromosome is highly homologous to the terR sites of the R6K plasmid. Cell. 55:467-475.

Hidaka, M., T. Kobayashi, Y. Ishimi, M. Seki, T. Enomoto, M. Abdel-Monem, and T. Horiuchi. 1992. Termination complex in Escherichia coli inhibits SV40 DNA replication in vitro by impeding the action of T antigen helicase. $\mathrm{J}$ Biol Chem. 267:5361-5365.

Hill, T.M., M.L. Tecklenburg, A.J. Pelletier, and P.L. Kuempel. 1989. tus, the trans-acting gene required for termination of DNA replication in Escherichia coli, encodes a DNA-binding protein. Proc Natl Acad Sci U S A. 86:1593-1597.

Hojgaard, A., H. Szerlong, C. Tabor, and P. Kuempel. 1999. Norfloxacin-induced DNA cleavage occurs at the dif resolvase locus in Escherichia coli and is the result of interaction with topoisomerase IV. Mol Microbiol. 33:1027-1036.

Hong, X., G.W. Cadwell, and T. Kogoma. 1995. Escherichia coli RecG and RecA proteins in R-loop formation. EMBO J. 14:2385-2392.

Ivanova, D., T. Taylor, S.L. Smith, J.U. Dimude, A.L. Upton, M.M. Mehrjouy, O. Skovgaard, D.J. Sherratt, R. Retkute, and C.J. Rudolph. 2015. Shaping the landscape of the Escherichia coli chromosome: replication-transcription encounters in cells with an ectopic replication origin. Nucleic Acids Res. 43:78657877.

Jameson, K.H., and A.J. Wilkinson. 2017. Control of Initiation of DNA Replication in Bacillus subtilis and Escherichia coli. Genes (Basel). 8.

Jin, D.J., C. Cagliero, and Y.N. Zhou. 2012. Growth rate regulation in Escherichia coli. FEMS Microbiol Rev. 36:269-287.

Kaimer, C., J.E. Gonzalez-Pastor, and P.L. Graumann. 2009. SpoIIIE and a novel type of DNA translocase, SftA, couple chromosome segregation with cell division in Bacillus subtilis. Mol Microbiol. 74:810-825.

Kaimer, C., K. Schenk, and P.L. Graumann. 2011. Two DNA translocases synergistically affect chromosome dimer resolution in Bacillus subtilis. J Bacteriol. 193:13341340 . 
Kamada, K., T. Horiuchi, K. Ohsumi, N. Shimamoto, and K. Morikawa. 1996a. Structure of a replication-terminator protein complexed with DNA. Nature. 383:598-603.

Kamada, K., K. Ohsumi, T. Horiuchi, N. Shimamoto, and K. Morikawa. 1996b. Crystallization and preliminary X-ray analysis of the Escherichia coli replication terminator protein complexed with DNA. Proteins. 24:402-403.

Kim, N., and S. Jinks-Robertson. 2012. Transcription as a source of genome instability. Nat Rev Genet. 13:204-214.

Krabbe, M., J. Zabielski, R. Bernander, and K. Nordstrom. 1997. Inactivation of the replication-termination system affects the replication mode and causes unstable maintenance of plasmid R1. Mol Microbiol. 24:723-735.

Kralicek, A.V., N.A. Vesper, G.B. Ralston, R.G. Wake, and G.F. King. 1993. Symmetry and secondary structure of the replication terminator protein of Bacillus subtilis: sedimentation equilibrium and circular dichroic, infrared, and NMR spectroscopic studies. Biochemistry. 32:10216-10223.

Kralicek, A.V., P.K. Wilson, G.B. Ralston, R.G. Wake, and G.F. King. 1997. Reorganization of terminator DNA upon binding replication terminator protein: implications for the functional replication fork arrest complex. Nucleic Acids Res. 25:590-596.

Kreuzer, K.N., and N.R. Cozzarelli. 1979. Escherichia coli mutants thermosensitive for deoxyribonucleic acid gyrase subunit $A$ : effects on deoxyribonucleic acid replication, transcription, and bacteriophage growth. J Bacteriol. 140:424-435.

Kuempel, P.L., J.M. Henson, L. Dircks, M. Tecklenburg, and D.F. Lim. 1991. dif, a recAindependent recombination site in the terminus region of the chromosome of Escherichia coli. New Biol. 3:799-811.

Kurth, I., and M. O'Donnell. 2009. Replisome Dynamics during Chromosome Duplication. EcoSal Plus. 3.

Langley, D.B., M.T. Smith, P.J. Lewis, and R.G. Wake. 1993. Protein-nucleoside contacts in the interaction between the replication terminator protein of Bacillus subtilis and the DNA terminator. Mol Microbiol. 10:771-779.

Larsen, N.B., I.D. Hickson, and H.W. Mankouri. 2014. Tus-Ter as a tool to study sitespecific DNA replication perturbation in eukaryotes. Cell Cycle. 13:2994-2998.

Lau, I.F., S.R. Filipe, B. Soballe, O.A. Okstad, F.X. Barre, and D.J. Sherratt. 2003. Spatial and temporal organization of replicating Escherichia coli chromosomes. Mol Microbiol. 49:731-743.

Lee, E.H., and A. Kornberg. 1992. Features of replication fork blockage by the Escherichia coli terminus-binding protein. J Biol Chem. 267:8778-8784.

Lee, E.H., A. Kornberg, M. Hidaka, T. Kobayashi, and T. Horiuchi. 1989. Escherichia coli replication termination protein impedes the action of helicases. Proc Natl Acad Sci U S A. 86:9104-9108.

Lemon, K.P., I. Kurtser, and A.D. Grossman. 2001. Effects of replication termination mutants on chromosome partitioning in Bacillus subtilis. Proc Natl Acad Sci U S A. 98:212-217.

Lesterlin, C., F.X. Barre, and F. Cornet. 2004. Genetic recombination and the cell cycle: what we have learned from chromosome dimers. Mol Microbiol. 54:1151-1160.

Lewis, P.J., G.B. Ralston, R.I. Christopherson, and R.G. Wake. 1990. Identification of the replication terminator protein binding sites in the terminus region of the Bacillus subtilis chromosome and stoichiometry of the binding. J Mol Biol. 214:73-84.

Lloyd, R.G., and C.J. Rudolph. 2016. 25 years on and no end in sight: a perspective on the role of RecG protein. Curr Genet. 62:827-840.

Maduike, N.Z., A.K. Tehranchi, J.D. Wang, and K.N. Kreuzer. 2014. Replication of the Escherichia coli chromosome in RNase HI-deficient cells: multiple initiation regions and fork dynamics. Mol Microbiol. 91:39-56.

Manna, A.C., K.S. Pai, D.E. Bussiere, C. Davies, S.W. White, and D. Bastia. 1996. Helicase-contrahelicase interaction and the mechanism of termination of DNA replication. Cell. 87:881-891. 
Marians, K.J., H. Hiasa, D.R. Kim, and C.S. McHenry. 1998. Role of the core DNA polymerase III subunits at the replication fork. Alpha is the only subunit required for processive replication. J Biol Chem. 273:2452-2457.

Markovitz, A. 2005. A new in vivo termination function for DNA polymerase I of Escherichia coli K12. Mol Microbiol. 55:1867-1882.

Matzke, A.J., B. Huettel, J. van der Winden, and M. Matzke. 2005. Use of two-color fluorescence-tagged transgenes to study interphase chromosomes in living plants. Plant physiology. 139:1586-1596.

McGlynn, P., and C.P. Guy. 2008. Replication forks blocked by protein-DNA complexes have limited stability in vitro. J Mol Biol. 381:249-255.

McGlynn, P., R.G. Lloyd, and K.J. Marians. 2001. Formation of Holliday junctions by regression of nascent DNA in intermediates containing stalled replication forks: RecG stimulates regression even when the DNA is negatively supercoiled. Proc Natl Acad Sci U S A. 98:8235-8240.

McGlynn, P., N.J. Savery, and M.S. Dillingham. 2012. The conflict between DNA replication and transcription. Mol Microbiol. 85:12-20.

McLean, M.J., K.H. Wolfe, and K.M. Devine. 1998. Base composition skews, replication orientation, and gene orientation in 12 prokaryote genomes. J Mol Evol. 47:691696.

Meijer, W.J., M. Smith, R.G. Wake, A.L. de Boer, G. Venema, and S. Bron. 1996. Identification and characterization of a novel type of replication terminator with bidirectional activity on the Bacillus subtilis theta plasmid pLS20. Mol Microbiol. 19:1295-1306.

Merrikh, H., Y. Zhang, A.D. Grossman, and J.D. Wang. 2012. Replication-transcription conflicts in bacteria. Nat Rev Microbiol. 10:449-458.

Mettrick, K.A., and I. Grainge. 2016. Stability of blocked replication forks in vivo. Nucleic Acids Res. 44:657-668.

Midgley-Smith, S.L., J.U. Dimude, T. Taylor, N.M. Forrester, A.L. Upton, R.G. Lloyd, and C.J. Rudolph. 2018. Chromosomal over-replication in Escherichia coli recG cells is triggered by replication fork fusion and amplified if replichore symmetry is disturbed. Nucleic Acids Res.

Mulcair, M.D., P.M. Schaeffer, A.J. Oakley, H.F. Cross, C. Neylon, T.M. Hill, and N.E. Dixon. 2006. A molecular mousetrap determines polarity of termination of DNA replication in E. coli. Cell. 125:1309-1319.

Mulugu, S., A. Potnis, Shamsuzzaman, J. Taylor, K. Alexander, and D. Bastia. 2001. Mechanism of termination of DNA replication of Escherichia coli involves helicasecontrahelicase interaction. Proc Natl Acad Sci U S A. 98:9569-9574.

Naqvi, A., E. Tinsley, and S.A. Khan. 2003. Purification and Characterization of the PcrA Helicase of Bacillus anthracis. Journal of Bacteriology. 185:6633-6639.

Natarajan, S., W.L. Kelley, and D. Bastia. 1991. Replication terminator protein of Escherichia coli is a transcriptional repressor of its own synthesis. Proc Natl Acad Sci U S A. 88:3867-3871.

Neylon, C., A.V. Kralicek, T.M. Hill, and N.E. Dixon. 2005. Replication termination in Escherichia coli: structure and antihelicase activity of the Tus-Ter complex. Microbiol Mol Biol Rev. 69:501-526.

Nordstrom, K. 2006. Plasmid R1--replication and its control. Plasmid. 55:1-26.

Pai, K.S., D.E. Bussiere, F. Wang, C.A. Hutchison, 3rd, S.W. White, and D. Bastia. 1996. The structure and function of the replication terminator protein of Bacillus subtilis: identification of the 'winged helix' DNA-binding domain. EMBO J. 15:3164-3173.

Pandey, M., M.M. Elshenawy, S. Jergic, M. Takahashi, N.E. Dixon, S.M. Hamdan, and S.S. Patel. 2015. Two mechanisms coordinate replication termination by the Escherichia coli Tus-Ter complex. Nucleic Acids Res. 43:5924-5935.

Payne, B.T., I.C. van Knippenberg, H. Bell, S.R. Filipe, D.J. Sherratt, and P. McGlynn. 2006. Replication fork blockage by transcription factor-DNA complexes in Escherichia coli. Nucleic Acids Res. 34:5194-5202. 
Peebles, C.L., N.P. Higgins, K.N. Kreuzer, A. Morrison, P.O. Brown, A. Sugino, and N.R. Cozzarelli. 1979. Structure and activities of Escherichia coli DNA gyrase. Cold Spring Harb Symp Quant Biol. 43 Pt 1:41-52.

Petit, M.A., and D. Ehrlich. 2002. Essential bacterial helicases that counteract the toxicity of recombination proteins. EMBO J. 21:3137-3147.

Possoz, C., S.R. Filipe, I. Grainge, and D.J. Sherratt. 2006. Tracking of controlled Escherichia coli replication fork stalling and restart at repressor-bound DNA in vivo. EMBO J. 25:2596-2604.

Reyes-Lamothe, R., E. Nicolas, and D.J. Sherratt. 2012. Chromosome replication and segregation in bacteria. Annu Rev Genet. 46:121-143.

Roecklein, B., A. Pelletier, and P. Kuempel. 1991. The tus gene of Escherichia coli: autoregulation, analysis of flanking sequences and identification of a complementary system in Salmonella typhimurium. Research in microbiology. 142:169-175.

Roecklein, B.A., and P.L. Kuempel. 1992. In vivo characterization of tus gene expression in Escherichia coli. Mol Microbiol. 6:1655-1661.

Rudolph, C.J., P. Dhillon, T. Moore, and R.G. Lloyd. 2007. Avoiding and resolving conflicts between DNA replication and transcription. DNA Repair (Amst). 6:981993.

Rudolph, C.J., A.A. Mahdi, A.L. Upton, and R.G. Lloyd. 2010a. RecG protein and singlestrand DNA exonucleases avoid cell lethality associated with PriA helicase activity in Escherichia coli. Genetics. 186:473-492.

Rudolph, C.J., A.L. Upton, G.S. Briggs, and R.G. Lloyd. 2010b. Is RecG a general guardian of the bacterial genome? DNA Repair (Amst). 9:210-223.

Rudolph, C.J., A.L. Upton, and R.G. Lloyd. 2009. Replication fork collisions cause pathological chromosomal amplification in cells lacking RecG DNA translocase. Mol Microbiol. 74:940-955.

Rudolph, C.J., A.L. Upton, A. Stockum, C.A. Nieduszynski, and R.G. Lloyd. 2013. Avoiding chromosome pathology when replication forks collide. Nature. 500:608611.

Samson, R.Y., Y. Xu, C. Gadelha, T.A. Stone, J.N. Faqiri, D. Li, N. Qin, F. Pu, Y.X. Liang, Q. She, and S.D. Bell. 2013. Specificity and function of archaeal DNA replication initiator proteins. Cell Rep. 3:485-496.

Smith, M.T., C.J. de Vries, D.B. Langley, G.F. King, and R.G. Wake. 1996. The Bacillus subtilis DNA replication terminator. J Mol Biol. 260:54-69.

Smith, M.T., and R.G. Wake. 1992. Definition and polarity of action of DNA replication terminators in Bacillus subtilis. J Mol Biol. 227:648-657.

Srivatsan, A., A. Tehranchi, D.M. MacAlpine, and J.D. Wang. 2010. Co-orientation of replication and transcription preserves genome integrity. PLOS Genet. 6:e1000810.

Straight, A.F., A.S. Belmont, C.C. Robinett, and A.W. Murray. 1996. GFP tagging of budding yeast chromosomes reveals that protein-protein interactions can mediate sister chromatid cohesion. Current biology : CB. 6:1599-1608.

Tanaka, T., and H. Masai. 2006. Stabilization of a stalled replication fork by concerted actions of two helicases. J Biol Chem. 281:3484-3493.

Tomasetti, C., L. Li, and B. Vogelstein. 2017. Stem cell divisions, somatic mutations, cancer etiology, and cancer prevention. Science. 355:1330-1334.

Trautinger, B.W., R.P. Jaktaji, E. Rusakova, and R.G. Lloyd. 2005. RNA polymerase modulators and DNA repair activities resolve conflicts between DNA replication and transcription. Mol Cell. 19:247-258.

Upton, A.L., J.I. Grove, A.A. Mahdi, G.S. Briggs, D.S. Milner, C.J. Rudolph, and R.G. Lloyd. 2014. Cellular location and activity of Escherichia coli RecG proteins shed light on the function of its structurally unresolved C-terminus. Nucleic Acids Res. 42:5702-5714.

Vivian, J.P., C.J. Porter, J.A. Wilce, and M.C. Wilce. 2007. An asymmetric structure of the Bacillus subtilis replication terminator protein in complex with DNA. J Mol Biol. 370:481-491. 
Wake, R., and G. King. 1997. A tale of two terminators: crystal structures sharpen the debate on DNA replication fork arrest mechanisms. Structure (London, England : 1993). 5:1-5.

Wake, R.G. 1996. DNA replication. Tussle with a terminator. Nature. 383:582-583.

Wang, J.D., M.B. Berkmen, and A.D. Grossman. 2007. Genome-wide coorientation of replication and transcription reduces adverse effects on replication in Bacillus subtilis. Proc Natl Acad Sci U S A. 104:5608-5613.

Wang, X., C. Lesterlin, R. Reyes-Lamothe, G. Ball, and D.J. Sherratt. 2011. Replication and segregation of an Escherichia coli chromosome with two replication origins. Proc Natl Acad Sci U S A. 108:E243-250.

Wendel, B.M., C.T. Courcelle, and J. Courcelle. 2014. Completion of DNA replication in Escherichia coli. Proc Natl Acad Sci U S A. 111:16454-16459.

Wilce, J.A., J.P. Vivian, A.F. Hastings, G. Otting, R.H. Folmer, I.G. Duggin, R.G. Wake, and M.C. Wilce. 2001. Structure of the RTP-DNA complex and the mechanism of polar replication fork arrest. Nat Struct Biol. 8:206-210.

Willis, N.A., G. Chandramouly, B. Huang, A. Kwok, C. Follonier, C. Deng, and R. Scully. 2014. BRCA1 controls homologous recombination at Tus/Ter-stalled mammalian replication forks. Nature. 510:556-559.

Zaritsky, A., and C.L. Woldringh. 2015. Chromosome replication, cell growth, division and shape: a personal perspective. Front Microbiol. 6:756.

Zhang, J., A.A. Mahdi, G.S. Briggs, and R.G. Lloyd. 2010. Promoting and avoiding recombination: contrasting activities of the Escherichia coli RuvABC Holliday junction resolvase and RecG DNA translocase. Genetics. 185:23-37.

\section{Further Reading}

\section{Figure Legends}

Figure 1. Arrangement of DNA replication terminators in the circular chromosomes of (A) Escherichia coli and (C) Bacillus subtilis. The two replication forks generated at the origin (oriC) move in opposite directions along the DNA and eventually approach one other and fuse within the terminus region diametrically opposed to oriC. The terminus region constitutes a replication fork trap in which the DNA terminators (denoted Ter) are arranged as two opposed groups, with the red terminators oriented to block movement of the clockwise replication fork and the blue terminators oriented to block the anticlockwise fork. Letters and roman numbers define Ter sites ( $A$ indicates the location of Ter $A$ in $E$. coli; $I$ indicates the location of TerI in B. subtilis). The STer region of the B. subtilis chromosome contains additional terminator sites used only during the stringent response. The chromosomal locations for the origin, the dif chromosome dimer resolution site and the genes for the terminator proteins, Tus (terminus utilization substance) in E. coli and RTP (replication terminator protein) in B. subtilis, are marked. The location of $r r n$ operons, which are highly transcribed particularly under fast growth conditions, are shown by green arrows, with the arrow pointing in the direction in which transcribing RNA polymerase molecules travel. (B) $\&$ (D) show consensus sequences for the E. coli Ter core sequence and the B. subtilis terminators. For B. subtilis the overlapping A and B sites are indicated.

Figure 2. Crystal structures of Terminator protein-DNA complexes. (A) Two crystal structures of the Tus-Ter complex of E. coli indicating the blocking and permissive ends of the complex (left, PDB 2i05), and in the "locked" conformation with DNA unwound at the blocking end and the C6 base of Ter DNA bound to its specific binding pocket in Tus (right, 
PDB 2i06), which contributes significantly to the fork arrest activity of the complex. (B) Crystal structure of an RTP dimer in complex with the high-affinity half of TerI (the B site) (PDB 2efw). RTP can form a symmetric dimer in solution and recognizes the partial DNA sequence symmetry in each half site that makes up each functional Ter site in B. subtilis. However, the partial asymmetry of each half site causes the RTP monomers to adopt somewhat different conformations in the half-site complex ("wing-up" conformation, on the left-hand monomer, and "wing-down" conformation on the right-hand monomer as viewed), and this might play a specific role in establishing cooperativity of binding to the second lowaffinity half-site (the A site), and optimizing contact with the oncoming replisome for its arrest. The cooperative binding of two dimers to each Ter site is essential for fork arrest activity. In the full complex, forks would be arrested when approaching from the right in the image shown. The structural basis for cooperative binding and how the whole complex interacts with the replisome are unknown features of interest.

Figure 3. Chromosome replication and cell growth in cells with one or two replication origins in the presence and absence of a replication fork trap. a) In the presence of a block to one replication fork on its way from oriC to the termination area the chromosome will remain under-replicated, as the second fork will be blocked by the Ter/Tus complexes in the termination area. b) Schematic representation of the replichore arrangement of an E. coli chromosome with an ectopic replication origin termed oriZ in the presence of a functional replication fork trap. oriZ indicates the integration of a duplication of the oriC sequence near the lacZYA operon (Wang et al., 2011). Directionality of replication and fork fusion locations are indicated by green arrows. c) Replichore parameters in the termination area of E. coli cells with two replication origins in the absence of a functional replication fork trap $(\Delta t u s)$. If forks escaping the termination area proceed with a speed similar to forks coming from oriC, then the fusion point should be in the location indicated by the blue arrows. If forks escaping the termination area are slowed by an increased number of replication-transcription conflicts, then forks should fuse closer to the termination area, as indicated by the grey arrows. The experimental observation is, however, that the fork fusion point is located closer to oriC (green arrows), indicating that forks escaping the termination area potentially encounter fewer problems than forks coming from oriC (Ivanova et al., 2015). d) Schematic representation of the replichore arrangement of an $E$. coli chromosome replicating exclusively from an ectopic replication origin in the presence (d i) and absence (d ii) of a functional replication fork trap. Directionality of replication and approximate fork fusion locations are indicated by green and blue arrows in the presence and absence of a functional fork trap $(\Delta t u s)$, respectively.

\section{Figure 4. Over-replication in the termination area in the absence of RecG helicase}

a) Replication profiles of $E$. coli cells in exponential phase. The number of reads (normalised against the reads for a stationary wild type control) is plotted against the chromosomal coordinate. Positions of oriC (green line) and primary Ter sites are shown above the plotted data with red and blue lines representing the left and right replichore as depicted in Figure 4A. The termination area between the innermost Ter sites is highlighted in light blue. Data were re-plotted from Rudolph et al. (2013). b) Growth of a $\Delta r e c G \Delta t u s$ rpo* strain in which the entire oriC region is deleted. c) Marker frequency analysis of a $\Delta r e c G \Delta t u s ~ r p o *$ strain that carries a temperature-sensitive allele of the main replication initiator protein DnaA. The strain was grown at $42^{\circ} \mathrm{C}$ to inactivate DnaA(ts) and therefore prevent oriC from being active. Data were re-plotted from Rudolph et al. (2013). d) Marker frequency analysis of chromosome replication in a double origin strain in the presence and absence of RecG. Strains were grown at $37^{\circ} \mathrm{C}$. 
Figure 5. Schematic illustrating how replication fork fusions might trigger over-replication in 2 the termination area and how this is normally prevented by proteins such as RecG and/or 3' 3 exonucleases. Note that the formation of a 3' flap can occur at both forks. However, for 4 simplicity the schematic shows only one such reaction. See text for further details. 
Cross-references to other eLS articles: As eLS is continually growing, authors writing update articles should check the validity of any cross references and add new cross references as appropriate. This is an intrinsic strength of the eLS format. In order to create a cross-reference please cite the other article's unique DOI and place it at the end of the relevant paragraph. As shown in the following example:

"The reversal potential defines the potential at which the net movement of charged ions into and out of the channel is zero, and for glutamate-activated channels is approximately $0 \mathrm{mV}$, whereas that for GABA-activated channels it is typically around -70 mV. See also: DOI: 10.1002/9780470015902.a0005907"

To identify appropriate eLS articles to cross-reference, go to www.els. net where abstracts, key concepts, keywords and figures for eLS articles are open to all browsers for quick reference. You can browse by topic and narrow by sub-topic, or enter text searches to track down related articles.

To find an article's DOI and build it in to your manuscript as a cross-reference, click on the article title. On the article landing page, the DOI is listed under the title and author information in the top left of the screen.

References and Further Reading: eLS articles may contain up to 50 cited References and up to 10 uncited Further Reading items. Both sections must be present. If important new papers have been published, they should be cited at relevant locations in the article text and listed in the References section. If textbooks, reference works, etc. were included in the Further Reading section, they should be updated with the latest editions.

FIGURES/TABLES: In your submission e-mail, please indicate those figures and tables that have been altered and those, including legends, that remain entirely unchanged from the previous version. We can then alert our typesetters accordingly.

To facilitate peer review, all NEW OR MODIFIED FIGURES AND TABLES, should be placed at the end of the manuscript file. Please do not embed them in the body text of your article. Our typesetters will take responsibility for inserting each figure or table as close as possible to your text citation (e.g. "See Figure 1....."as seen in Table 2"). Please ensure that you cite all figures and tables in the text.

NEW OR MODIFIED FIGURES (though not tables) should also be supplied separately in high resolution, editable figure files. We recommend EPS files for line illustrations. PDF, PPT, DOC, AI, CDR, WMF and INDD files can also be used; provided they are in vector format (i.e. still editable). TIFF files should only be used for unlabelled, unedited photographs.

Line illustrations supplied electronically should not have a resolution less than $600 \mathrm{dpi}$. Tone illustrations (black and white and colour photographs for instance) should have a resolution of around $300 \mathrm{dpi}$. Please do NOT supply 'thumbnails' or low resolution images).

Figure and Table Permissions: We must remind you that it is the author's responsibility to obtain permission to reproduce any figures or tables that have been published elsewhere (note this only applies to new figures or tables, you do not need to seek permission to re-use figures or tables from the current version of this eLS article). 
Please note that even if you have modified a copyrighted figure or table, it is still likely to require permission.

To help with the applications process, we have provided you with a permissions request form, attached to your Author Guidelines email.

Please confirm at the bottom of your manuscript the numbers of the figures or tables that do not require permission. 
(A)

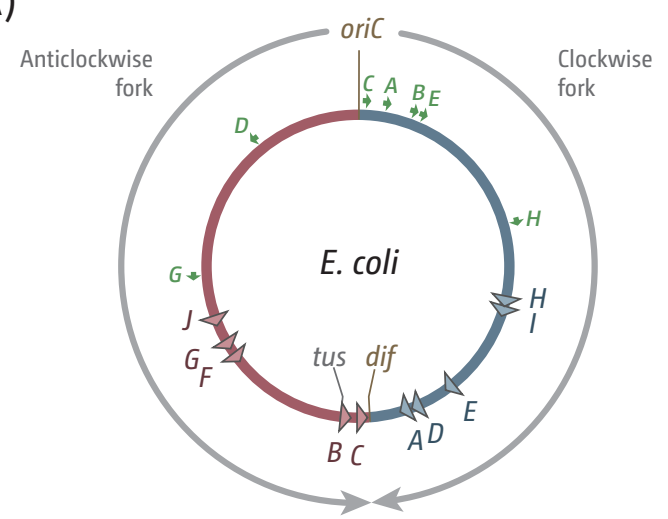

(B) E. coli Ter consensus sequence (central core)

$$
{ }_{G}^{6}{ }_{G}^{T} A_{A}^{T} \operatorname{GTTGTAAC}_{G}^{\mathrm{T}} \mathrm{A}
$$

(C)

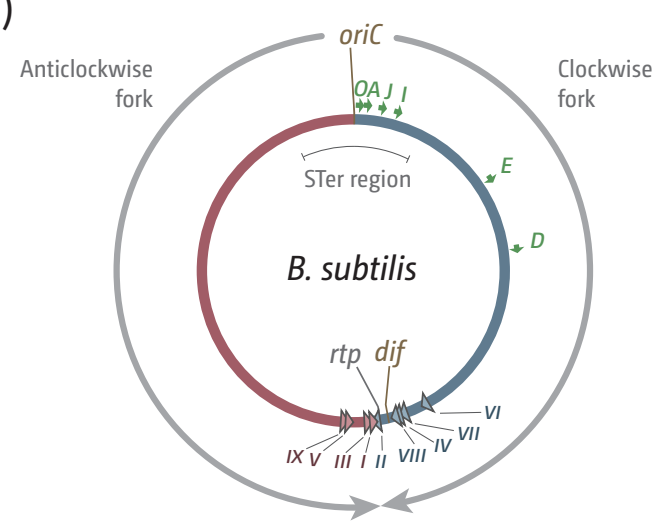

(D) B. subtilis ter consensus sequence:

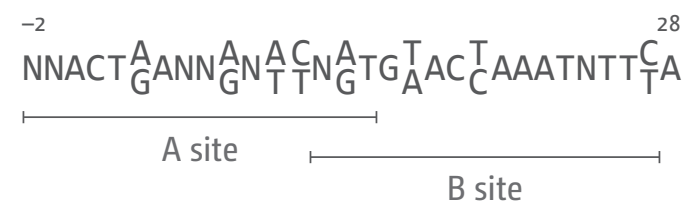


(A) E. coli Tus bound to TerA DNA
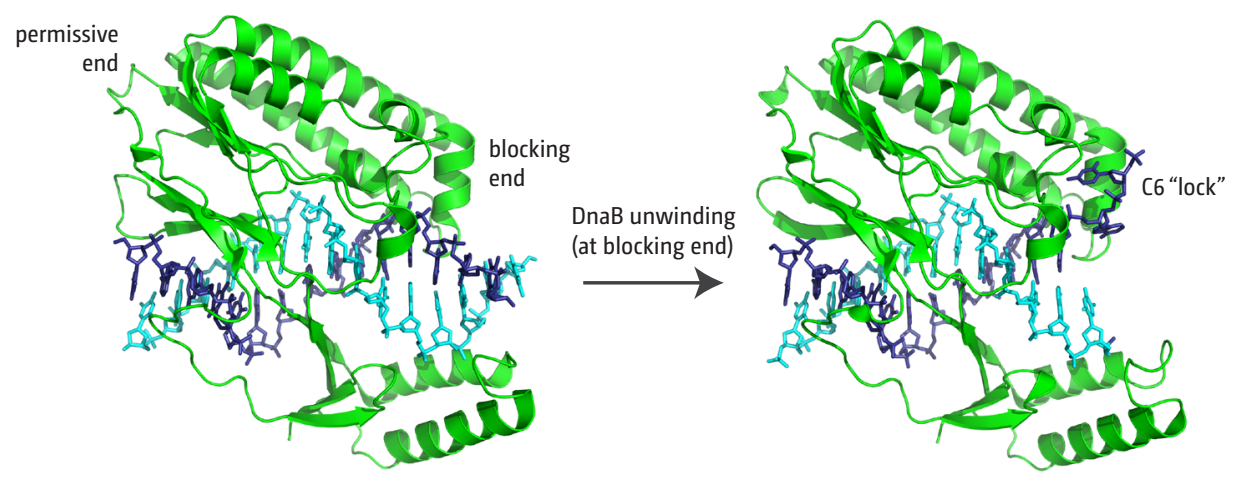

(B) B. subtilis RTP dimer bound to a Terl half-site DNA (the B-site)

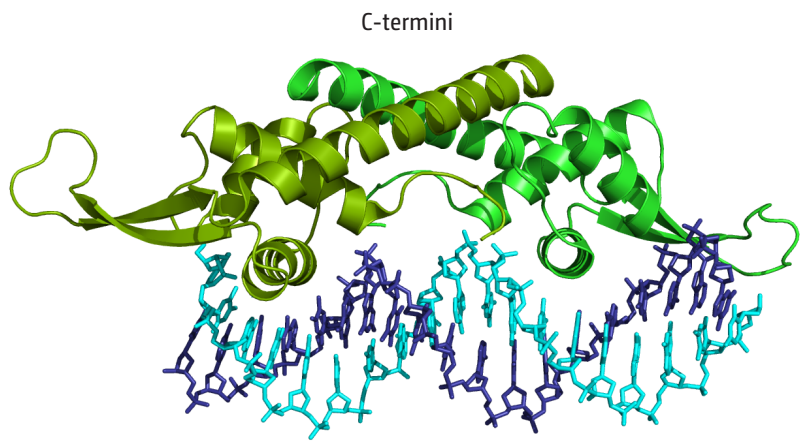


(A)

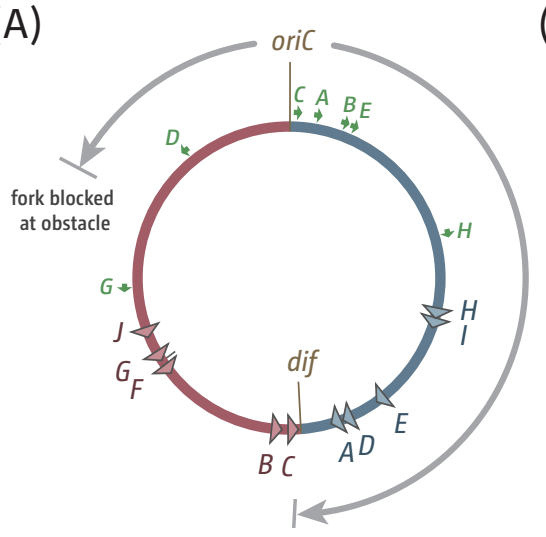

(B)

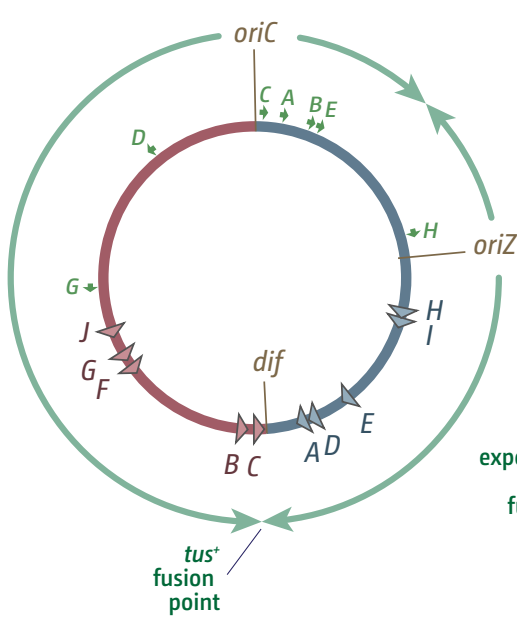

(C)

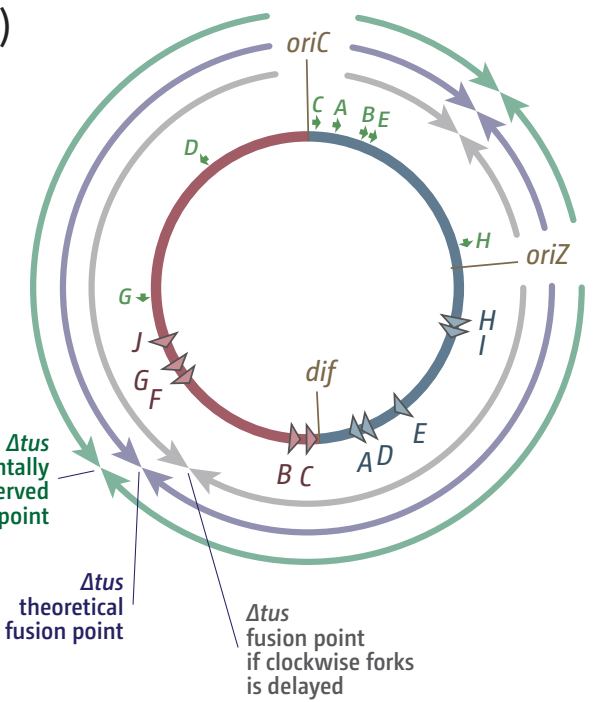

(D I)

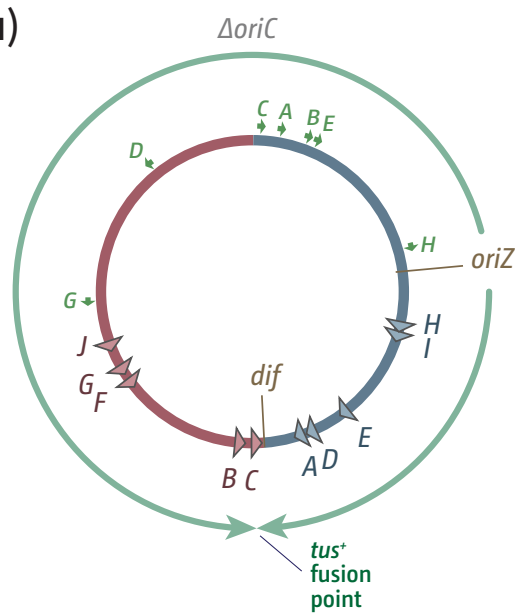

(D II)

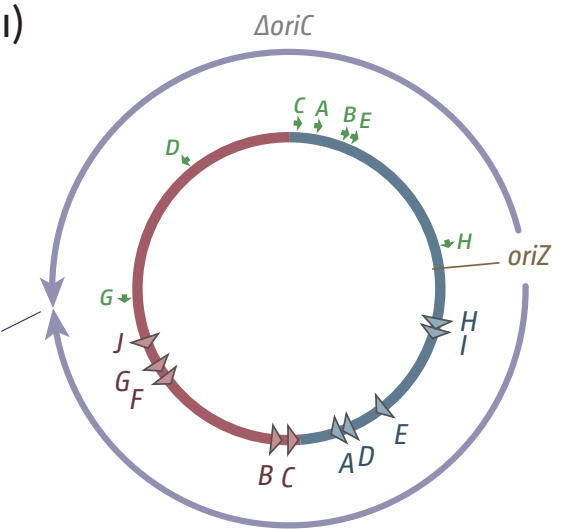


A

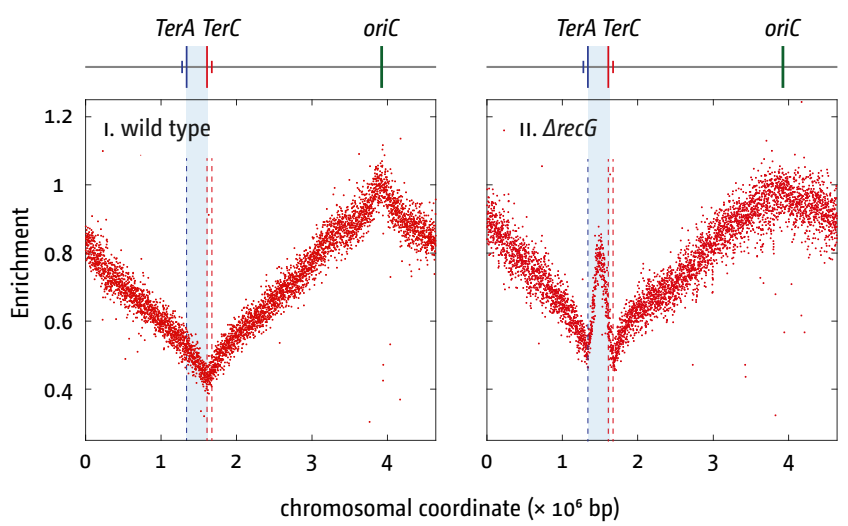

B

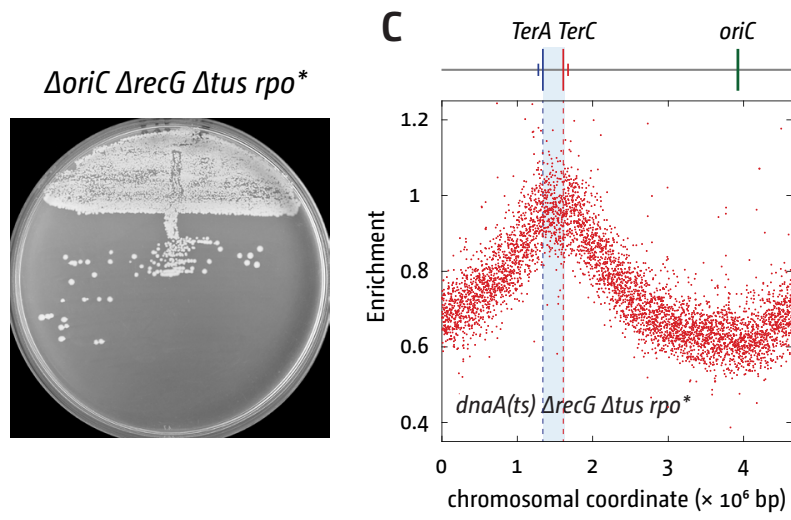

D

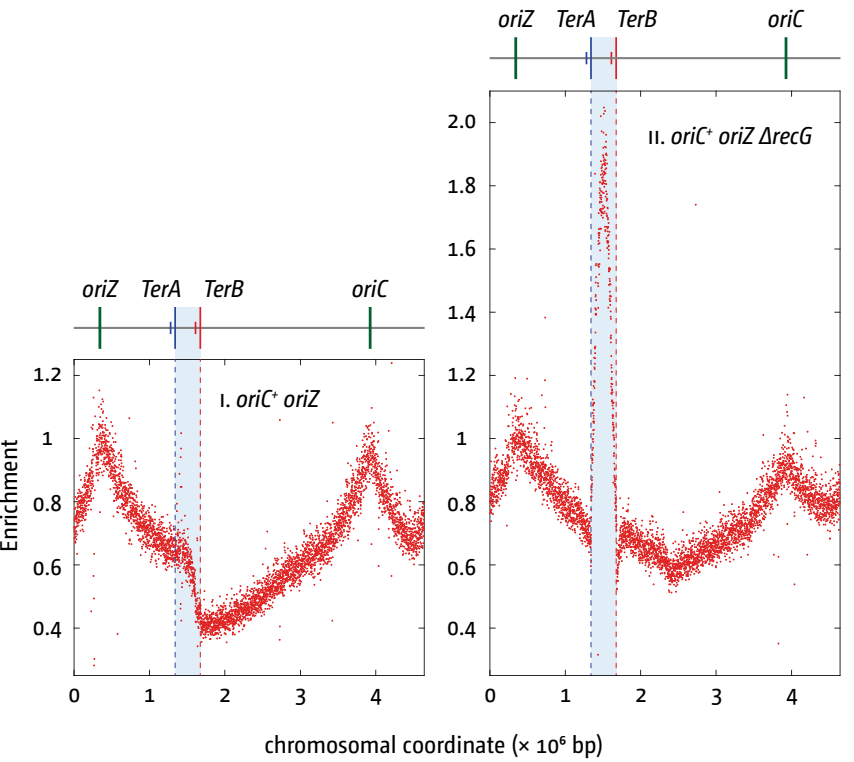

Rudolph et al. Figure 4 
(A I) converging replication

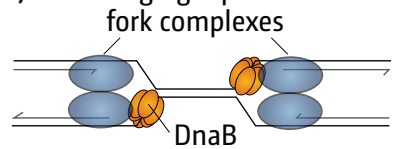

(B)

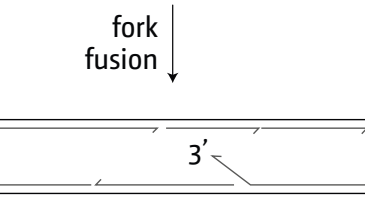

(C)

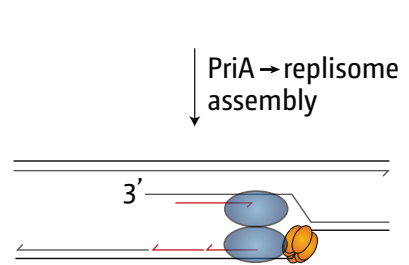

RecBCD $\rightarrow$ PriA $\rightarrow$ replisome recombination $\downarrow$ assembly

(D)

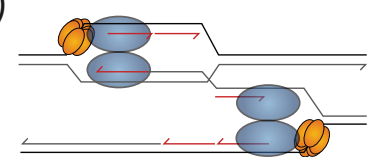

(A II)

termination

degradation by 3 ' exonucleases

RecG $\rightarrow$ conversion

to 5 ' flap and degradation

by 5 ' exonucleases 